\title{
Bond percolation in higher dimensions
}

\author{
Eric I. Corwin* \\ Department of Physics, University of Oregon, Eugene, Oregon 97403, USA
}

Robin Stinchcombe ${ }^{\dagger}$

Rudolf Peierls Centre for Theoretical Physics, University of Oxford, 1 Keble Road, Oxford OX1 3NP, United Kingdom

\author{
M. F. Thorpe \\ Department of Physics, Arizona State University, Tempe, Arizona 85287-1604, USA \\ and Rudolf Peierls Centre for Theoretical Physics, University of Oxford, 1 Keble Road, Oxford OX1 3NP, United Kingdom
}

(Received 11 April 2013; published 3 July 2013)

\begin{abstract}
We collect results for bond percolation on various lattices from two to fourteen dimensions that, in the limit of large dimension $d$ or number of neighbors $z$, smoothly approach a randomly diluted Erdős-Rényi graph. We include results on bond-diluted hypersphere packs in up to nine dimensions, which show the mean coordination, excess kurtosis, and skewness evolving smoothly with dimension towards the Erdős-Rényi limit.
\end{abstract}

DOI: 10.1103/PhysRevE.88.014102

PACS number(s): 64.60.ah, 61.43.Bn, 81.05.Rm

\section{INTRODUCTION}

Percolation theory [1,2] asks if there is a connected path across a system. Examples are water percolating through ground coffee beans and forest fires spreading from tree to tree.

Although this is an old subject, interest continues, including in higher dimensions, where rigorous bounds on $p_{c}$ (the critical concentration) have recently been established [3]. We denote by $z$ the number of initial bonds at any site of a particular regular lattice (e.g., triangular net and simple cubic) before bond dilution occurs $(p=1)$. It is convenient to define the mean coordination $\langle r\rangle$ at the percolation point as

$$
\langle r\rangle=z p_{c},
$$

which facilitates the comparison between various lattices in various dimensions as the mean coordination $\langle r\rangle$ at percolation varies much less than $p_{c}$ itself. A very simple argument suggests that $\langle r\rangle=2$ at the transition as each site must have one bond entering and one bond leaving to form a connected pathway. While this is the most efficient scenario, it does not happen quite this way in a random system for two reasons. First, there is redundancy where there is more than one connection between two points, leading to a loop. Loops push the mean coordination $\langle r\rangle$ above 2 , because at least two sites with coordination 3 must be involved in forming a loop. Second, there is irrelevancy where dead ends and isolated regions are formed that would not carry a current if the bonds were wires in a conducting network. Irrelevancy pushes the mean coordination $\langle r\rangle$ below 2, as some sites are singly coordinated. Both these situations are illustrated in Fig. 1. We will see that there is a tendency for the effects of redundancy and irrelevancy to cancel making $\langle r\rangle=2$ a reasonable starting approximation for low dimensions $d$ and/or low initial coordination $z$. However, for very high dimension

\footnotetext{
*eric.corwin@gmail.com

${ }^{\dagger}$ r.stinchcombe1@physics.ox.ac.uk

${ }^{\ddagger} \mathrm{mft} @$ asu.edu
}

$d$ or initial coordination $z$, the mean coordination number $\langle r\rangle$ approaches unity because of the preponderance of dangling bonds. The result $\langle r\rangle=2$ at the transition can also be derived by Maxwell-type constraint counting [4] of the number of floppy modes [5,6] or residual degrees of freedom $f$ in the system.

Connectivity percolation can be regarded as a special case ( $g=1$ ) of a larger class of problems having $g \geqslant 1$ degrees of freedom per site. An example would be vector displacements in two dimensions, where $g=2$. For $g \geqslant 2$ such problems are usually referred to as rigidity percolation $[5,6]$.

Maxwell constraint counting [4] is more usually employed in problems involving rigidity, but can also be applied to connectivity percolation problems as a special case, with $g=1$. More generally, there are $g$ degrees of freedom associated with each site and $z$ constraints are present (the number of bonds at each site is assumed to be exactly $z$ everywhere initially) with probability $p$, so that

$$
f=g-z p / 2 \text {, }
$$

which goes to zero at $p_{c}=2 g / z$ and hence gives the result $\langle r\rangle=2 g$ at percolation. Note that the number of floppy modes is not exactly zero at the transition as fluctuations in local coordination number allow for local redundancy and irrelevancy, but nevertheless it has been shown that the number of floppy modes at the transition is extremely low [7], making $\langle r\rangle=2 g$ an unusually accurate approximation for $g \geqslant 2$ (typically within $1 \%$ ). For example, in the case of rigidity percolation of a triangular net under bond dilution Maxwell counting gives a result of $\langle r\rangle=4$, while numerical simulations [7] find $\langle r\rangle=3.961 \pm 0.002$, which is very close to, but clearly less than, 4. However, the constraint counting result $\langle r\rangle=2$ for connectivity percolation gets worse in higher dimensions in which $\langle r\rangle=1$ is reached.

\section{BETHE LATTICE}

A useful universal guideline is provided by the Bethe lattice, which is a treelike network that contains no loops [8,9]. For a connected path there must be one way in from a previous layer 


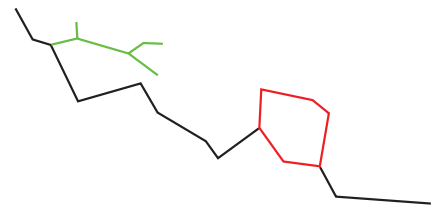

FIG. 1. (Color online) Connected path in black across part of a sample with redundancy via the red (dark gray) loop, which is overconstrained with one redundant bond, and irrelevancy via the green (light gray) region, which contains dangling ends that are not involved in percolation.

and one of the remaining $z-1$ ways out must be occupied, so that $p_{c}=1 /(z-1)$, a result that can be rigorously found [8]. Hence the mean coordination $\langle r\rangle$ at percolation is given by

$$
\langle r\rangle=z p_{c}=z /(z-1) .
$$

There is a binomial distribution of local coordination numbers due to the random dilution, so the probability of a site having $r$ bonds present out of a total of $z$ possible is given by

$$
P(r)=\sum_{r=0}^{z}\left(\begin{array}{l}
z \\
r
\end{array}\right) p^{r}(1-p)^{z-r}
$$

and hence the $n$th moment $\left\langle r^{n}\right\rangle$ is given by

$$
\left\langle r^{n}\right\rangle=\sum_{r=0}^{z} r^{n} P(r),
$$

leading to the mean coordination

$$
\langle r\rangle=z p
$$

and the square of the width $\Delta r$ given by

$$
(\Delta r)^{2}=\left\langle r^{2}\right\rangle-\langle r\rangle^{2}=z p(1-p) .
$$

For the Bethe lattices at the percolation threshold, this width becomes

$$
\Delta r=\frac{\sqrt{z(z-2)}}{(z-1)} .
$$

A particularly interesting limit is large $z \rightarrow \infty$, where we obtain what we will refer to as the Erdős-Rényi limit, reached when percolation occurs upon bond dilution in a graph that initially has $N$ nodes, each one connected to every other node [10] as $N \rightarrow \infty$. In this limit

$$
\langle r\rangle=\Delta r=1 .
$$

In the limit that the number of nodes goes to infinity, the chance of finding a loop becomes infinitesimally small and hence the large- $z$ Bethe lattice result is obtained. An example of a finite Erdôs-Rényi graph [10] is shown in Fig. 2.

\section{A UNIVERSAL PLOT}

It is convenient to combine all results for bond percolation on various lattices as a plot of the mean coordination $\langle r\rangle$ against the width of the distribution $\Delta r$, which is shown in Fig. 3. The results for the two-dimensional (2D), 3D, and hypercubic lattices are conveniently summarized with original references in Ref. [11]. The two-dimensional results, shown

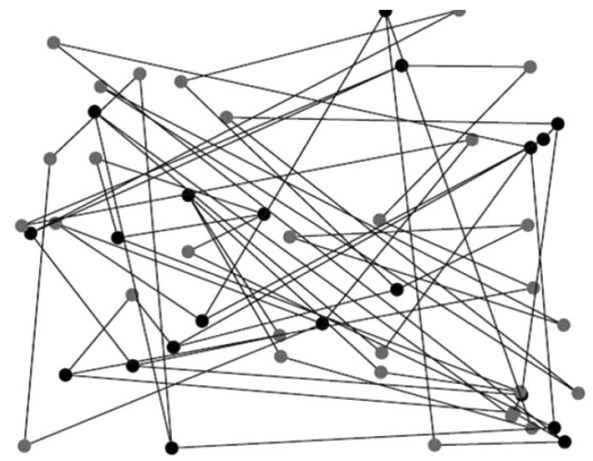

FIG. 2. Bond diluted finite Erdős-Rényi graph, where before dilution every node was connected to every other node.

in red in Fig. 3, are from left to right, following the thin red line, honeycomb, kagome, square net, and triangular net. The general trend is higher initial coordination $z$ to the right going to lower initial coordination $z$ to the left, which tends to the isostatic point shown at $(0,2)$.

The three-dimensional results, shown in blue, are from left to right, following the thin blue line, diamond, simple cubic, body centered cubic, and face centered cubic, with the latter two close together but following the general trend with higher initial coordination $z$ to the right going to lower initial coordination $z$ to the left, which again tends to the isostatic point at $(0,2)$. Also included in Fig. 3 are the results

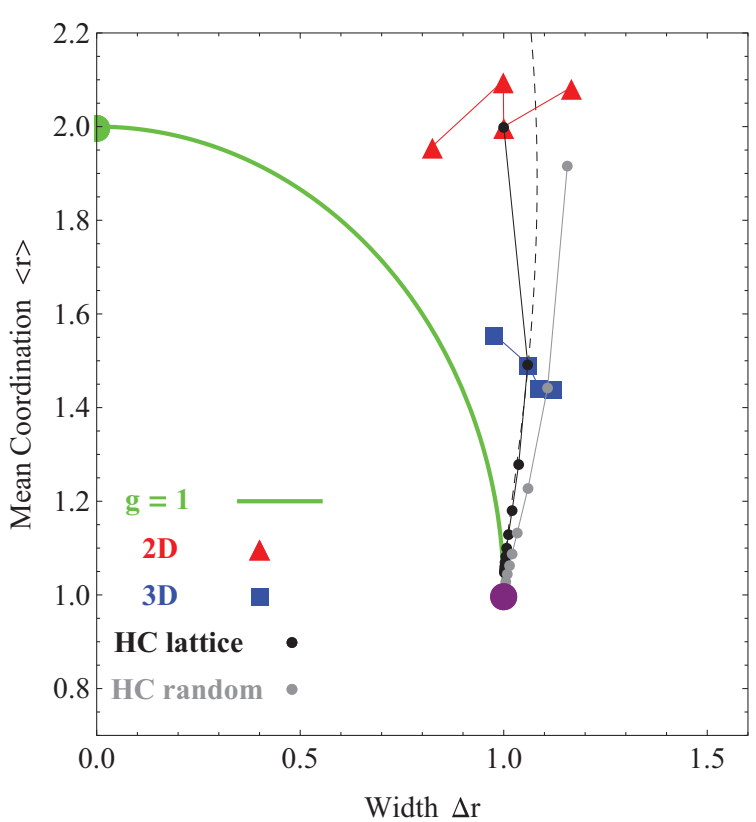

FIG. 3. (Color online) Results for the mean coordination against the width for 2D lattices (red triangles), 3D lattices (blue squares), hypercubic lattices (black circles), and random hypersphere packings (gray circles) at the percolation threshold. The straight lines joining adjacent points are only for guidance of the eye. The thick green line is the Bethe lattice result with the isostatic point at $(0,2)$ and the Erdôs-Rényi result $(1,1)$ shown as the large purple dot. The dashed line shows the result of a $1 /(z-1)$ expansion [15] given in Eq. (11) for hypercubic lattices. 
for bond-diluted hypercubic lattices from $d=2$ up to $d=13$ where the mean coordination $\langle r\rangle$ is obtained from (1) and the width from (7).

The results for diluted noncrystalline hypersphere packings were obtained from computer simulations of jammed configurations of $N=262144$ monodisperse particles (in two dimensions a 50-50 mixture of bidisperse particles with size ratio 1.4:1 was used to avoid crystallization) as described in Ref. [12]. The particles interact with a harmonic contact potential defined as

$$
V(r)=\epsilon(\sigma-r)^{2} \Theta(\sigma-r),
$$

where $\sigma$ is the particle radius, $\epsilon$ the energy scale of the potential, and $r$ the distance between particles. Energy is minimized at a given packing fraction via either a conjugate gradient [13] or fast inertial relaxation engine [14] minimization technique. Starting from a random configuration at a density well above jamming and given two values of packing fraction that bracket the jamming transition density, the jamming point is found via a golden mean bisection search. Jamming is identified as the packing fraction corresponding to the onset of nonzero energy as derived from the potential (10). For the purposes of percolation studies, two hyperspheres are said to be connected neighbors if there is a nonzero overlap between them. We find that the values of $\langle r\rangle$ and $\Delta r$ are rather insensitive to a (small) distance from the jamming transition.

Note that both sets of high-dimensional results, for bond percolation in hypercubic lattices and random hypersphere packings, approach the Erdős-Rényi limit, as can be seen from Fig. 3. The highest dimension explored of $d=13$ for hypercubic lattices and $d=9$ for random hypersphere packings are already very close to the point $(1,1)$. This is because loops become less important in higher dimensions, discussed next.

If we consider the mean and width of the percolation variable $r$ then the Erdôs-Rényi limit is [from Eqs. (3)-(9)] the same as the $z \rightarrow \infty$ limit of the Bethe lattice (tree) result. The tree is in turn the loopless limit of a general lattice and from simple geometric path counting considerations, the loopless limit is the large- $z$ and, equivalently, the large- $d$ limit of a general lattice. The probability of two sites being joined by a graph with $n$ links will be proportional to $p_{c}^{n}$. Now consider all graphs with $n$ steps. For the trees we have $r=n / 2$ and for all other graphs with a partial or full loop $r>n / 2$. The key observation is that in high dimensions, $p$ goes like $1 / d$, as can be seen for the Bethe lattice in Eq. (8). For example, hypercubic lattices have $z=2 d$ and for random packings $z$ is even larger. Therefore, as $d \rightarrow \infty$ those diagrams with $r=n / 2$ overwhelmingly dominate and hence only the trees contribute and the Erdős-Rényi limit is reached. While this is not a formal proof, it demonstrates the plausibility of the result and should form the basis for a formal mathematical proof.

For completeness, we include the results of Gaunt and Ruskin [15], who performed a $1 /(z-1)$ expansion for bond percolation on bond-diluted hypercubic lattices where $z=2 d$ and found that percolation occurs at

$$
p_{c}=\sigma\left(1+\frac{5}{2} \sigma^{2}+\frac{15}{2} \sigma^{3}+57 \sigma^{4}+\cdots\right),
$$

where $\sigma=(z-1)^{-1}$. Note that the leading term is the Bethe lattice result. We include this result in Fig. 3 as a dashed line, which is seen to be very close indeed to the results of numerical simulations (black dots) for hypercubic lattices with $d \geqslant 3$, then deviating at $d=2$ for the square lattice.

Another convenient way to monitor the approach of dilute hypercubic lattices to the Erdôs-Rényi limit is to track the skewness $\gamma_{1}$ and excess kurtosis $\gamma_{2}$, which respectively monitor the evolution of the asymmetry and the deviation from Gaussian behavior of the distribution of contacts (for a Gaussian distribution $\gamma_{1}=\gamma_{2}=0$ ). These are defined in terms of the moments of the distribution as

$$
\begin{gathered}
\gamma_{1}=\frac{\left\langle(r-\langle r\rangle)^{3}\right\rangle}{\left\langle(r-\langle r\rangle)^{2}\right\rangle^{3 / 2}}, \\
\gamma_{2}=\frac{\left\langle(r-\langle r\rangle)^{4}\right\rangle}{\left\langle(r-\langle r\rangle)^{2}\right\rangle^{2}}-3 .
\end{gathered}
$$

For Bethe lattices they take the values

$$
\begin{gathered}
\gamma_{1}=\frac{3-2\langle r\rangle}{\sqrt{\langle r\rangle(2-\langle r\rangle)}}, \\
\gamma_{2}=\frac{1}{\langle r\rangle(2-\langle r\rangle)}-\frac{6(\langle r\rangle-1)}{\langle r\rangle} .
\end{gathered}
$$

These are plotted as the solid lines in Fig. 4. In the limit of a Bethe lattice with large $z$, the distribution of coordination number becomes a Poisson distribution with $p(r)=e^{-1} / r$ ! and thus $\langle r\rangle=\Delta r=\gamma_{1}=\gamma_{2}=1$. Note that for the Bethe lattice, the skewness goes through zero at $\langle r\rangle=3 / 2$, which corresponds to $z=3$, and the excess kurtosis goes through zeros at $\langle r\rangle=(9 \pm \sqrt{3}) 6=1.211$ and 1.789, which correspond to $z=4 \pm \sqrt{3}=2.227$ and 6.928 , respectively.

The skewness and the excess kurtosis for the hypercubic lattices can be obtained for the known values of $p_{c}$ from Ref. [11] and using Eqs. (4) and (5), respectively. For the binomial distribution, the skewness is

$$
\gamma_{1}=\frac{1-2 p}{\sqrt{z p(1-p)}}
$$

and the excess kurtosis is

$$
\gamma_{2}=\frac{1}{z p(1-p)}-\frac{6}{z}
$$

and these are also plotted at the percolation threshold in Fig. 4, which shows how they approach the Erdős-Rényi limit in high dimensions, providing further evidence of the relative unimportance of loops in connectivity percolation in higher dimensions. Results for the skewness and excess kurtosis can also be obtained from the expansion [15] given in Eq. (11), coupled with Eqs. (14) and (15), and are shown as the dashed lines in Fig. 4. Also shown in Fig. 4 are directly computed results for the skewness and excess kurtosis for bond-diluted random hypersphere packs at the percolation threshold in higher dimensions. Again a similar trend towards the Erdős-Rényi limit in high dimensions is very apparent. All results for bond-diluted hypersphere packings at the percolation threshold are tabulated in Table I.

These kinds of argument extend from percolation to a range of other processes. Among them are other $q$-state Potts models 


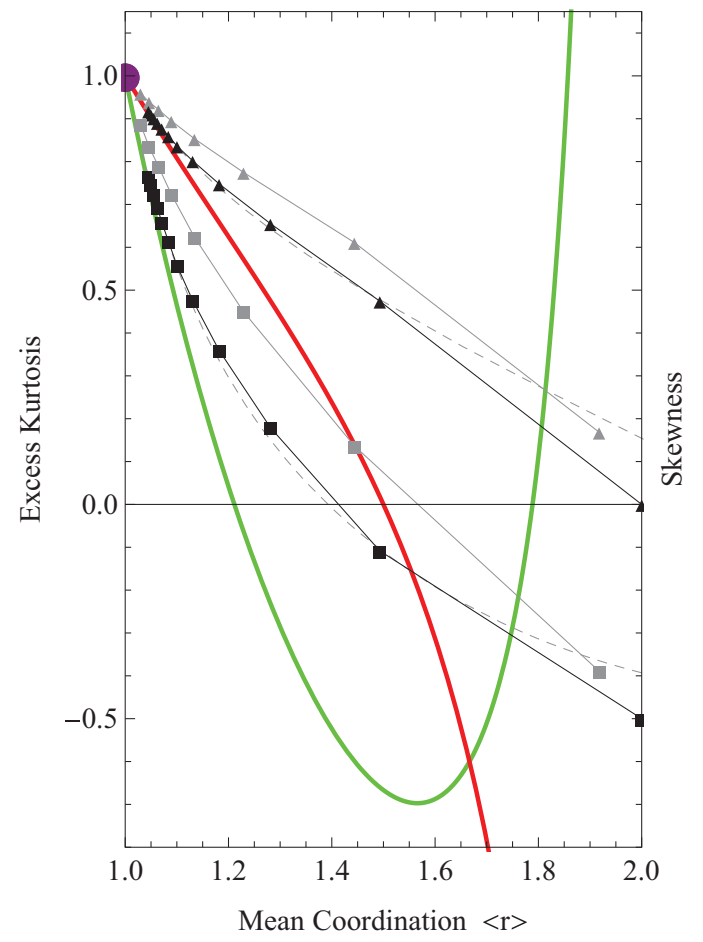

FIG. 4. (Color online) Skewness [thick red (dark gray) line] and excess kurtosis [thick green (light gray) line] as a function of the mean coordination $\langle r\rangle$ for Bethe lattices at the percolation threshold. Also shown are the skewness (triangles) and excess kurtosis (squares) for hypercubic lattices as gray symbols and random hypersphere packings as black symbols. The straight lines joining adjacent symbols are guides to the eye. The Erdős-Rényi result $(1,1)$ is shown as the large purple dot. The dashed line shows the result of a $1 /(z-1)$ expansion [15] given in Eq. (11).

(the $q \rightarrow 1$ limit is bond percolation [16]), which include the Ising model $(q=2)$. This was perhaps the first system for which small $1 / z$ was systematically exploited by Brout [17] and Englert [18]. The limit $1 / z \rightarrow 0$ gives mean field theory, associated with the tree graphs of the linked-cluster many-body theory. This is the starting point for a $1 / z$ expansion involving
TABLE I. Tabulated values for $\langle r\rangle, \Delta r$, skewness, and excess kurtosis for random hypersphere packings of $N=262144$ particles in dimensions $d=2-9$. Note that all packings are constructed with monodisperse spheres except for $d=2$, for which a 50-50 mixture of bidisperse particles with size ratio 1.4:1 is used.

\begin{tabular}{ccccc}
\hline \hline$d$ & $\langle r\rangle$ & $\Delta r$ & Skewness & Excess kurtosis \\
\hline 2 & 1.9174 & 1.1564 & 0.1687 & -0.3890 \\
3 & 1.4435 & 1.1079 & 0.6113 & 0.1368 \\
4 & 1.2289 & 1.0599 & 0.7749 & 0.4499 \\
5 & 1.1338 & 1.0335 & 0.8535 & 0.6242 \\
6 & 1.0890 & 1.0209 & 0.8954 & 0.7244 \\
7 & 1.0642 & 1.0145 & 0.9214 & 0.7891 \\
8 & 1.0459 & 1.0090 & 0.9397 & 0.8355 \\
9 & 1.0294 & 1.0052 & 0.9596 & 0.8877 \\
\hline \hline
\end{tabular}

graphs with increasing numbers of loops, which account for the fluctuation effects absent from mean field theory. It is interesting to note that arguments similar to those given here were previously given by Brout [17], who exploited the link between trees and mean field theory for the Ising model, using large $z$, where the factor $\left(J k_{B} T\right)^{n}$ in an $n$ th-order graph is analogous to the $p_{c}^{n}$ here. In the Ising model $J$ is the exchange interaction between spins and $T$ is the temperature.

\section{CONCLUSION}

We have shown that all bond-dilution results have universal features so that results for various lattices in various dimensions can be displayed on a single plot and these results approach the Erdős-Rényi limit in high dimensions.

\section{ACKNOWLEDGMENTS}

We would like to thank the US National Science Foundation for support under Career Awards No. DMR-1255370 (E.I.C.) and No. DMR-0703973 (M.F.T.) and by a Major Research Instrumentation grant, Office of Cyber Infrastructure, "MRIR2: Acquisition of an Applied Computational Instrument for Scientific Synthesis," Grant No. OCI-0960354.
[1] D. Stauffer and A. Aharony, Introduction to Percolation Theory (CRC, Boca Raton, 1994).

[2] J. W. Essam, Rep. Prog. Phys. 43, 833 (1980).

[3] S. Torquato and Y. Jiao, Phys. Rev. E 87, 032149 (2013).

[4] J. C. Maxwell, Philos. Mag. 27, 294 (1864).

[5] M. F. Thorpe, J. Non-Cryst. Solids 57, 355 (1983).

[6] M. F. Thorpe, in Encyclopedia of Complexity and Systems Science, edited by R. A. Meyers, Vol. 5 (Springer, New York, 2009), pp. 6013-6024.

[7] D. J. Jacobs and M. F. Thorpe, Phys. Rev. E 53, 3682 (1996).

[8] M. E. Fisher and J. W. Essam, J. Math. Phys. 2, 609 (1961).

[9] M. F. Thorpe, in Excitation in Disordered Systems, edited by M. F. Thorpe, NATO Advanced Study Institute, Series B: Physics, Vol. 78 (Plenum, New York, 1982), pp. 85-107.
[10] P. Erdős and A. Rényi, Publ. Math. Inst. Hung. Acad. Sci. 5, 17 (1960).

[11] http://en.wikipedia.org/wiki/Percolation-threshold

[12] P. Charbonneau, E. I. Corwin, G. Parisi, and F. Zamponi, Phys. Rev. Lett. 109, 205501 (2012).

[13] M. R. Hestenes and E. Stiefel, J. Res. Natl. Bur. Stand. 49, 409 (1952).

[14] E. Bitzek, P. Koskinen, F. Gähler, M. Moseler, and P. Gumbsch, Phys. Rev. Lett. 97, 170201 (2006).

[15] D. S. Gaunt and H. Ruskin, J. Phys. A: Math. Gen. 11, 1369 (1978).

[16] C. M. Fortuin and P. W. Kasteleyn, Physica 57, 536 (1972).

[17] R. H. Brout, Am. J. Phys. 34, 830 (1966).

[18] F. Englert, Phys. Rev. 129, 567 (1963). 